\title{
Transformation of alignment files improves performance of variant callers for long-read RNA sequencing data
}

Vladimir B. C. de Souza ${ }^{1}$, Ben T. Jordan², Elizabeth Tseng ${ }^{3}$, Elizabeth A. Nelson ${ }^{4}$, Karen K. Hirschi $^{4,5}$, Gloria Sheynkman ${ }^{2,6^{*}}$, Mark D. Robinson ${ }^{1^{*}}$

"Correspondence: gs9yr@virginia.edu; mark.robinson@mls.uzh.ch

${ }^{1}$ Department of Molecular Life Sciences and SIB Swiss Institute of Bioinformatics, University of Zurich, 8057 Zurich, Switzerland

${ }^{2}$ Department of Molecular Physiology and Biological Physics, University of Virginia School of Medicine, Charlottesville, VA 22903, USA

${ }^{3}$ PacBio, San Francisco, USA

${ }^{4}$ Department of Cell Biology and Cardiovascular Research Center, University of Virginia School of Medicine, Charlottesville, VA 22908, USA

${ }^{5}$ Department of Medicine, Yale University School of Medicine; Department of Genetics, Yale University School of Medicine; Yale Cardiovascular Research Center, Yale University School of Medicine, New Haven, CT 06511, USA

${ }^{6}$ Center for Public Health Genomics and UVA Cancer Center, University of Virginia, Charlottesville, VA 22908, USA

\section{Abstract}

Long-read RNA sequencing (IrRNA-seq) produces detailed information about full-length transcripts, including novel and sample-specific isoforms. Furthermore, there is opportunity to call variants encoded in the transcribed regions of genes directly from IrRNA-seq data. However, most state-of-the-art variant callers have been developed for genomic DNA and thus require modifications to call variants from IrRNA-seq data. Here, we benchmark variant callers GATK, DeepVariant, Clair3, and NanoCaller on PacBio IrRNA-seq, or "Iso-Seq", data. In particular, we found that careful processing of alignment files is critical to achieve better calling performance of indels and SNPs using DeepVariant and indels using Clair3. 


\section{Background}

The detection of genetic variants from next-generation sequencing (NGS) data remains of high interest for applications in clinical diagnostics and to improve our understanding of genetic diseases [1-3]. The most popular variant detection tools have been developed for short-read DNA sequencing data, including GATK [4], bcftools [5], FreeBayes [6] and Platypus [7], among others. However, since the short reads are typically not long enough to encompass multiple variants in a single read, they cannot be phased, i.e., co-associated to individual isoforms. Fortunately, with the increase in throughput and accuracy of long-read technologies, opportunities for detection of genetic variants from long reads are expanding. For example, IsoPhase [8] was developed to call and phase SNPs from Iso-Seq data, though it does not characterize insertions or deletions (indels). Such information linked to full-length reads offers the opportunity to predict open reading frames (ORFs) with variations that alter protein coding potential $[9,10]$ or transcriptional outcomes, including frame shifts (from indels), truncations or extensions (from altered stop codons), and disrupted splice sites [11]. However, such information is not incorporated in protein prediction. For example, when SQANTI [9] predicts ORFs from long reads, SNPs and indels are reverted to the sequence of the reference genome, losing potentially important patient-specific variations.

Several tools have been designed for calling variants from long reads of DNA aligned to a reference genome, including: DeepVariant [12], Clair3 [13], NanoCaller [14] (for SNP/indel calling); Longshot [15] (for SNP calling); PEPPER-Margin-DeepVariant [16] (for SNP/indel calling from nanopore sequencing data); pbsv [17] (for structural variant calling); and WhatsHap [18] (for variant phasing). However, it is also possible to call variants from IrRNA-seq alignments. For example, TAMA [19] calls variants directly from long reads aligned to a reference genome. Reference-free isoform clustering strategies exist, including IsoCon [20], where a "polishing" step is done to correct errors while keeping variants. Nevertheless, since isoform-clustering and reference-alignment approaches operate at per-isoform and per-gene coverage, respectively, isoform-level approaches tend to show lower sensitivity.

Here, we focus on calling genetic variants from IrRNA-seq reads. Specifically, we benchmark and incorporate existing tools that call variants from DNA-seq or short-read RNA-seq data. The GATK pipeline has already been repurposed to call SNPs and indels from short-read RNA-seq data by using the function SplitNCigarReads (SNCR) [4]. DeepVariant, Clair3 and NanoCaller use a deep learning (DL) approach in which variants are detected by analysis of read-alignment images; Clair3 uses a pileup model to call most variants, and a more computationally-intensive full-alignment model to handle more complex variants. All the DL-based tools have been trained and tested on long DNA sequencing reads, but not on IrRNA-seq data. In this work, we compare the performance of GATK, DeepVariant, Clair3 and NanoCaller to call variants from PacBio IrRNA-seq data (i.e., Iso-Seq data). We identify factors that influence variant calling performance, including read coverage, proximity to splice junctions, presence of homopolymers, and allele-specific expression. Finally, we present a pipeline to manipulate spliced alignments of SNCR-generated BAM files, such that files are suitable for variant calling. 


\section{Results and Discussion}

To call variants from IrRNA-seq alignments, we found that transformations of the BAM alignment encodings are critical. This is because while variant calling from aligned DNA sequences data involves analysis of contiguously aligned reads, variant calling from IrRNA-seq alignments must handle reads with gaps representing large intronic regions. For example, GATK employs the SNCR function to split reads at introns (Ns in their CIGAR string), thus converting a single isoform alignment into a set of reads representing distinct exons (Fig. 1A). However, SNCR also applies the primary-alignment flag to only one of the split reads and all others receive a supplementary-alignment flag, which can affect performance of downstream tools. Thus, we developed a pipeline, flagCorrection, to ensure all fragments retain the original flag (Fig. 1A; Fig. S1 shows an IGV screenshot).

To assess the performance of variant callers, we assembled a set of ground-truth variants for two datasets (Jurkat and WTC-11 cell lines) from Illumina DNA-seq data, retaining only variants from high confidence regions (see Methods) and for which there is sufficient corresponding IrRNA-seq coverage (numbers after filtering are shown in Fig. 1B).

We first evaluated the performance of DeepVariant. To measure the performance gains of DeepVariant calls from manipulated BAM files (Fig. 1A), we called variants from Jurkat and WTC-11 Iso-Seq datasets using three variations: DeepVariant alone, DeepVariant combined with SNCR (SNCR+DeepVariant), and DeepVariant combined with both SNCR and flagCorrection (SNCR+flagCorrection+DeepVariant). The precision and recall of each pipeline, separated by variant type (SNP or indel) and across various minimum Iso-Seq read coverage thresholds is shown in Fig. S2. DeepVariant alone had the lowest performance when read coverage is low, mainly because of low recall. However, when the coverage is high, DeepVariant exhibited similar performance to SNCR+flagCorrection+DeepVariant. This is because when the read coverage at a candidate variant site is high (i.e., typically in an exonic region), the number of intron-containing reads (so-called $\mathrm{N}$-cigar reads; Fig. $1 \mathrm{C}$ ) at that same site is typically low (Fig. S3). SNCR+DeepVariant showed low performance for all read coverage thresholds, highlighting the need for flagCorrection. Moreover, to directly illustrate how the performance of DeepVariant-based pipelines is affected by the presence of introns, we compare performance according to the proportion of $\mathrm{N}$-cigar reads (Fig. S4). Taken together, DeepVariant's recall is heavily dependent on the proportion of N-cigar reads, with extremely low recall when this proportion is high, and correct management (SNCR+flagCorrection) of alignment flags allows DeepVariant to maintain a high performance. 
bioRxiv preprint doi: https://doi.org/10.1101/2022.02.08.479579; this version posted February 10,2022 . The copyright holder for this preprint (which was not certified by peer review) is the author/funder, who has granted bioRxiv a license to display the preprint in perpetuity. It is made available under aCC-BY 4.0 International license.

A
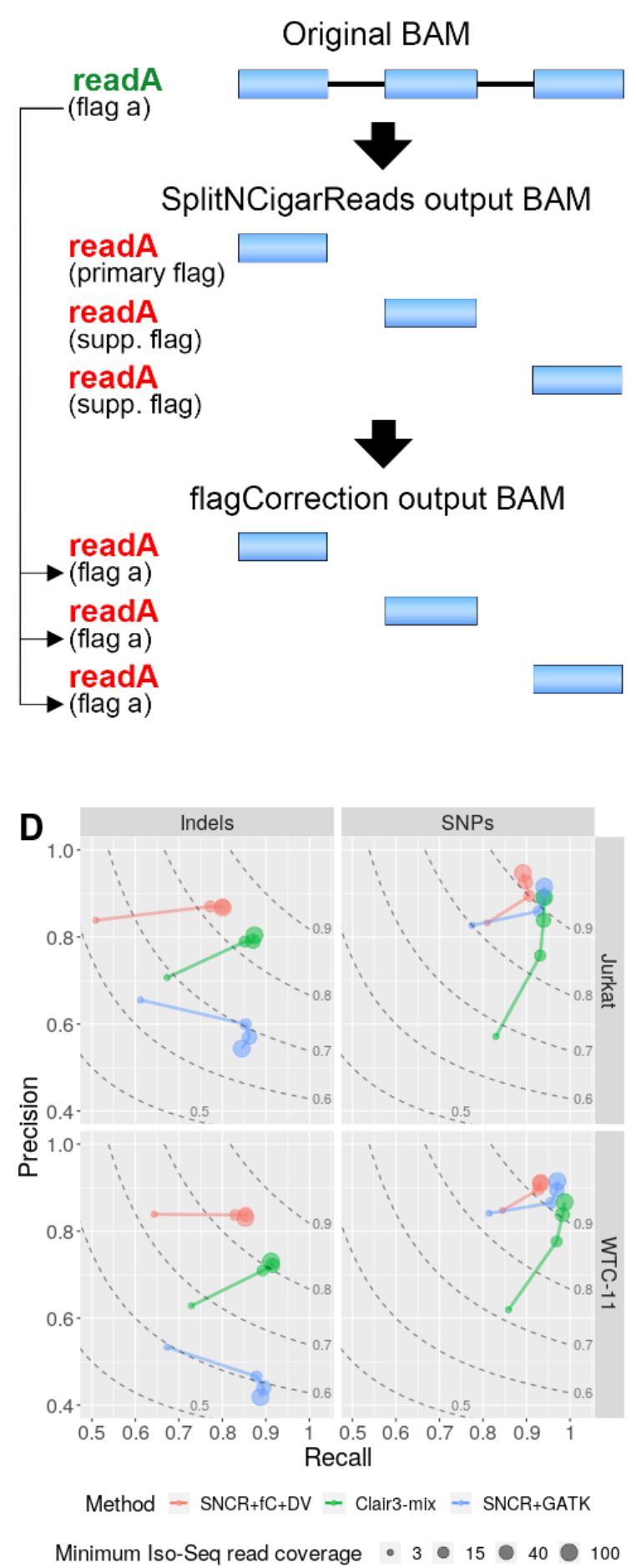

B

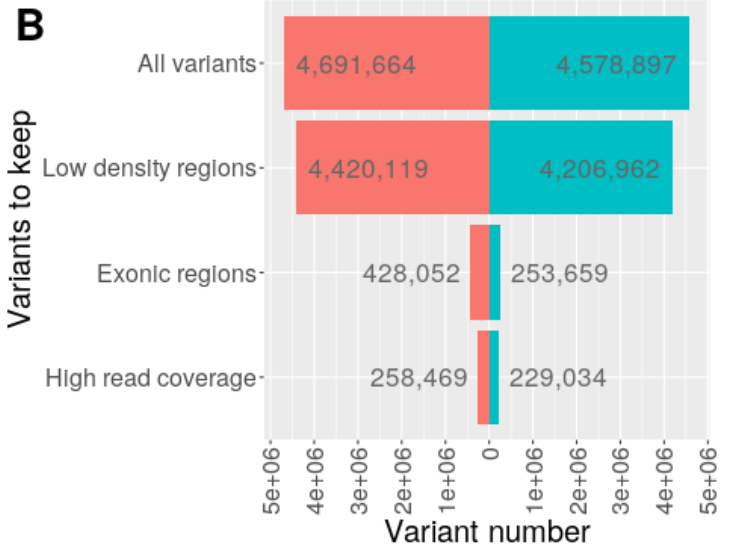

Dataset $\square$ Jurkat $\square$ WTC-11

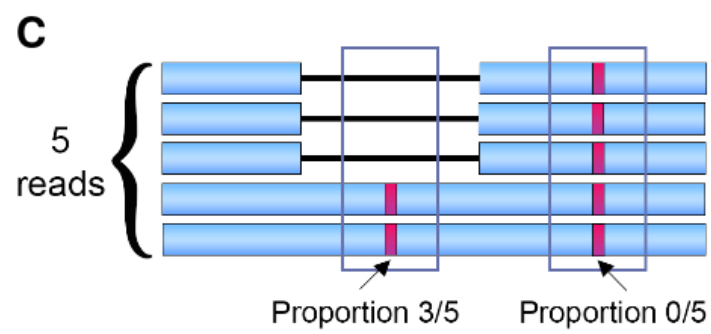

E

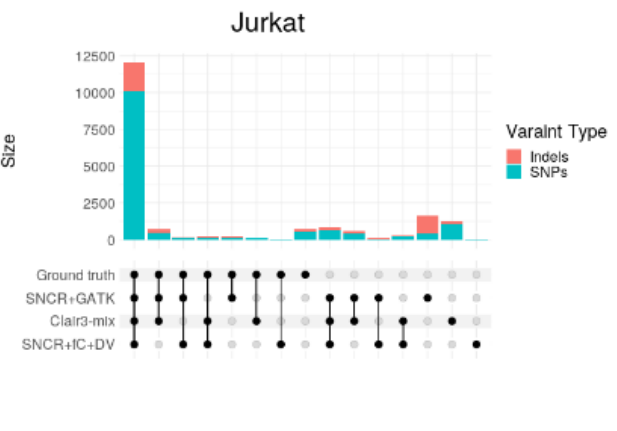

F

WTC-11

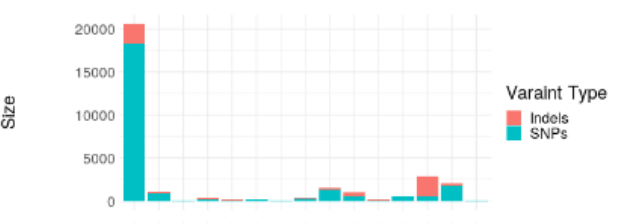

8
8.00
Set size

Fig. 1 Alignment file transformation for optimised calling of genetic variants from IrRNA-seq data and variant calling performance across the best pipelines on PacBio Iso-Seq reference datasets. (A) Alignment file (BAM) transformations to make spliced IrRNA-seq alignments suitable for variant calling. First, GATK's SNCR function is used to split the reads at Ns in their cigar string, such that exons become distinct reads. Second, GATK's flagCorrection function attributes the flag of the original read to all corresponding fragment reads. (B) The number of genetic variants kept in the ground-truth (Illumina DNA-seq) VCF files (for Jurkat and WTC-11 datasets) after filtering; $y$-axis refers to variant sites that are successively retained, as follows: All variants, all sites in the VCF files; Low density regions, sites residing in regions such that there is a maximum of 3 variants in a $201 \mathrm{bp}$ window; Exonic regions, sites where the Iso-Seq coverage is at least 1; High read coverage, sites where the short-read coverage is at least 20 and 72 for Jurkat and WTC-11, respectively; see Methods for more details. (C) Schematic 
with proportion of reads that contain introns (N-cigar reads) at two different variant sites (red boxes). (D) Precision-recall curves; point sizes indicate the filtering threshold for minimum read coverage; dashed lines represent F1-scores. "Clair3-mix" denotes using Clair3 to call SNPs and SNCR+flagCorrection+Clair3 to call indels. SNCR-SplitNCigarReads; fC-flagCorrection; DV-DeepVariant. (E,F) UpSet plots show the intersection of variants called by the pipelines with the ground truth for Jurkat (E) and WTC-11 (F) datasets; sites shown here were filtered according to a minimum Iso-Seq read coverage of 30 .

Next, we evaluated the performance of Clair3. Similarly to the DeepVariant comparisons, we compared Clair3's performance from unmodified BAMs to those subjected to SNCR and/or flagCorrection. Fig. S5 shows the performance of Clair3-based pipelines using variants merged from both pileup and full-alignment models (recommended by Clair3's developers [13]), and Fig. S6 shows variants called only by the pileup model. These results show that the full-alignment model could not accurately call variants from IrRNA-seq data. Moreover, although SNCR+flagCorrection+Clair3 on the pileup model increases indel calling precision while maintaining recall compared to Clair3 alone (Fig. S6), the full-alignment model causes many false positives (FPs). Thus, we decided to exclusively use the pileup model to call variants from IrRNA-seq data, and apply this strategy for all subsequent analyses here. Using the pileup model for SNP calling, SNCR+flagCorrection+Clair3 presented a slightly higher precision but decreased recall. Therefore, we suggest using SNCR+flagCorrection+Clair3 for indels and Clair3 for SNPs, hereafter referred to as "Clair3-mix".

Using the same strategy, we compared the performance of NanoCaller-based pipelines. NanoCaller and SNCR+flagCorrection+NanoCaller generally failed to call variants from Iso-Seq data (recall approximately to zero); despite SNCR+NanoCaller showing a higher recall, it is still much lower compared to the other pipelines (Fig. S7) and is therefore left out of subsequent comparisons.

To compare the performance of DeepVariant and Clair3 with (SNCR+)GATK, we selected the most accurate version of their pipelines found so far. Precision-recall curves are shown in Fig. 1D, split by variant type (indel and SNP). For indel calling, SNCR+flagCorrection+DeepVariant and Clair3-mix were the best pipelines (similar F1 scores; see Table S1). However, the DeepVariant-based pipeline showed higher precision, while Clair3-mix's recall was higher. SNCR+GATK showed very low precision to call indels. For SNP calling, all three methods showed similar performance at high coverage, but Clair3-mix showed lower precision at lower coverage. Taken together, SNCR+flagCorrection+DeepVariant was the best performing pipeline. Intersections of the called variants compared to the ground truth are shown in Fig. 1E-F. Notably, most of the true variants were called by all methods (true positives, TPs); a considerable number of variants were called by Clair3-mix and/or SNCR+GATK, but were absent from the ground truth (FPs); most FPs from SNCR+GATK are indels.

Next, we investigated factors that influence the performance of variant calling specifically from IrRNA-seq data. Variants situated close to splice junction boundaries could be more challenging to detect, especially for variant callers that process images of alignments. Thus, we determined variant calling performance according to splice junction proximity. Fig. 2A shows the precision, recall, and F1 scores for SNP sites with a minimum Iso-Seq coverage (20 reads or more). For a site to be considered near a splice junction, at least half of the reads that contain the site must contain the same splice junction and the site cannot be further than 20 base pairs (bp) away from that junction. For SNP calls near splice junctions, all pipelines showed a drop in recall but a slight increase in precision, indicating 
bioRxiv preprint doi: https://doi.org/10.1101/2022.02.08.479579; this version posted February 10, 2022. The copyright holder for this preprint (which was not certified by peer review) is the author/funder, who has granted bioRxiv a license to display the preprint in perpetuity. It is made available under aCC-BY 4.0 International license.

that variant calling was more conservative near junctions. However, SNCR+flagCorrection+DeepVariant tended to not detect SNPs near splice junctions, therefore showing a considerable drop in its F1 score. Clair3-mix was the least affected, with no apparent change in its F1 score. On the other hand, to call indels near splice junctions, all pipelines showed a similar drop in their F1 scores (Fig. 2B). Overall, variant calling is less reliable (especially for indel calling) near splice junctions, which could be partially explained by alignment issues near splice junctions due to the presence of these variants.
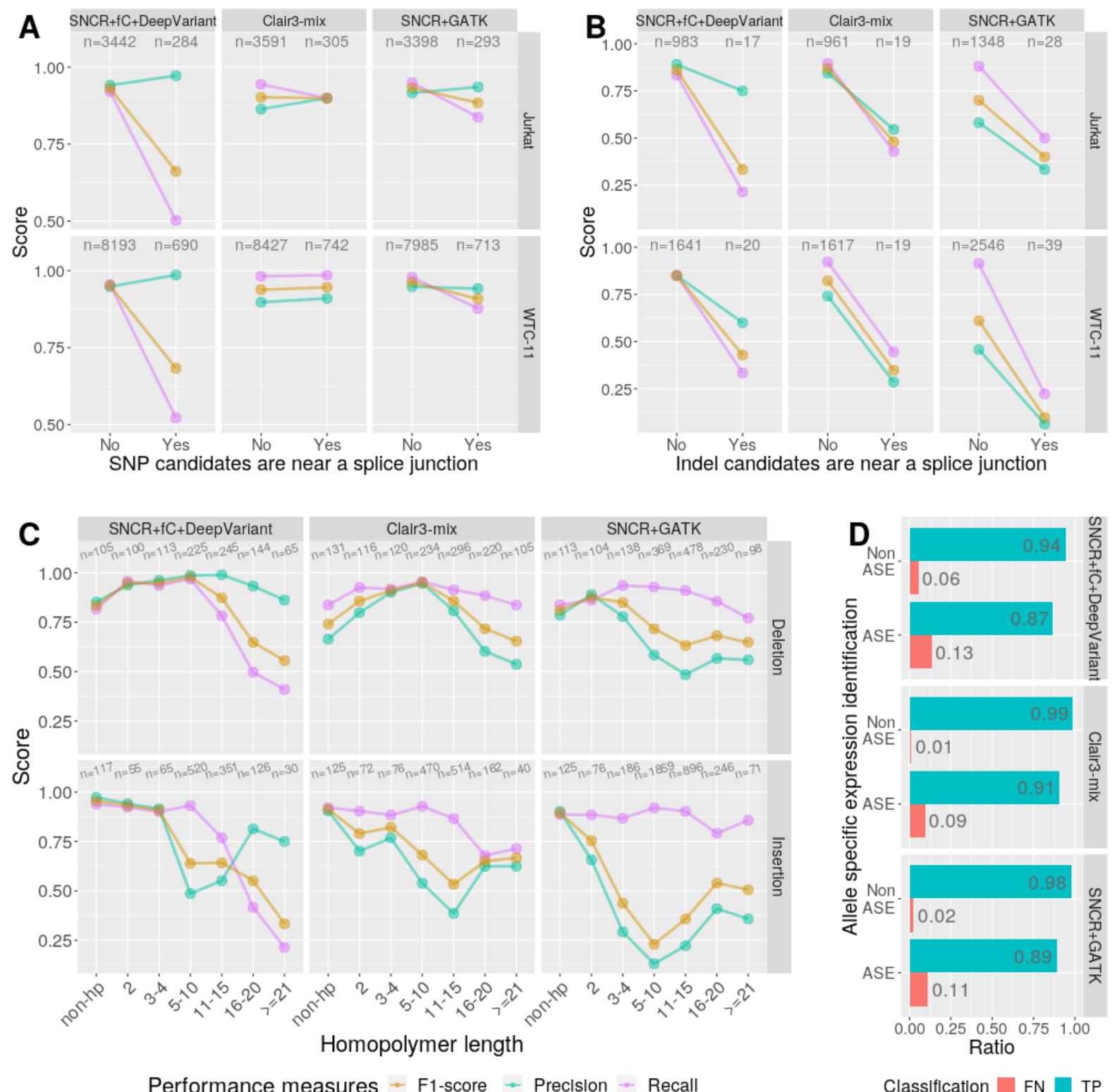

Fig. 2 Variant calling performance according to splice junction proximity, homopolymers or allele-specific expression. $n$ indicates the number of sites used to calculate each performance measure.

SNCR-SplitNCigarReads; fC-flagCorrection. In Clair3-based pipelines, only the pileup model was used. Performance measures for SNP (A) and indel (B) calling of sites far from (No) and near to (Yes) splice junctions for datasets Jurkat and WTC-11. (C) Performance measures of indel calling of sites in non-homopolymers (non-hp) and within homopolymers of specified length; results only from WTC-11 dataset. (D) FN and TP rates of heterozygous SNP calling from sites in allele-specific expressed (ASE) genes and non-ASE genes; results only from WTC-11 dataset; only sites with RNA short-read coverage of 40 and Iso-Seq read coverage of 20 were considered. 
Another factor that could influence variant calling is the presence of homopolymers. Since sequencing accuracy of long-read platforms is lower in homopolymer-containing regions [21], we evaluated methods to call indels within such regions from the WTC-11 dataset (Jurkat dataset not included due to lower read coverage). Fig. $2 \mathrm{C}$ shows how precision, recall and F1 score vary according to the length of homopolymer. Unsurprisingly, the performance of most pipelines dropped as the length of homopolymer increased; this drop was slightly sharper for insertions.

Since RNA-seq can only observe expressed variants and some genes express only one allele (allele-specific expression; ASE), we hypothesised that variants from ASE genes, corresponding to the lower abundance transcript, would be correlated with a higher false negative (FN; i.e., an undetected true variant) rate. To investigate this, we used Illumina RNA-seq short reads on WTC-11 cells to categorise heterozygous SNPs in the ground truth as either ASE or non-ASE sites (see Methods). Fig. 2D highlights that the proportion of FN to TP calls is higher at ASE genes compared to non-ASE genes (chi-squared-test of independence: $p$-value < 0.001). As expected, genes expressing a dominant allele do not give the opportunity to observe heterozygous sites, and may need to be considered in future workflows.

\section{Conclusions}

Our comparison of variant calling from IrRNA-seq data highlights that gapped alignments decrease performance of standard tools, but after appropriate treatment of alignments and read flags, a high performance can be recovered. In particular, the SplitNCigarReads and flagCorrection functions as applied to input BAM files enable an increase in recall of DeepVariant and the precision of Clair3's pileup model (for indel calling); Clair3-mix and SNCR+flagCorrection+DeepVariant are among the best-performing pipelines to call indels, the former having higher recall and the latter higher precision. For SNP calling, SNCR+GATK, SNCR+flagCorrection+DeepVariant and Clair3-mix showed similar performance, although Clair3-mix underperformed at lower read coverage. Our results show that when variants are near splice junctions, indel calling was less reliable, and SNCR+flagCorrection+DeepVariant's recall strongly drops for SNP calling in such regions. Moreover, the performance of all pipelines dropped for indels within homopolymer regions, and we confirmed that ASE genes are a blind spot for RNA-seq-based variant calling.

Overall, we have provided insights on how to call genetic variants from IrRNA-seq data, and we constructed a pipeline (https://github.com/vladimirsouza/lrRNAseqVariantCalling) for such analyses, which should also work with Oxford Nanopore IrRNA-seq data. This work should be of relevance for applications in genomic medicine, in which variants can be detected directly from IrRNA-seq data collected on patients. It would also be of interest for protein prediction workflows, since genetic variants must be taken into account to correctly predict ORFs and variant protein sequences. 


\section{Methods}

\section{PacBio Iso-Seq datasets}

PacBio IrRNA-seq data (i.e., Iso-Seq) was collected on both Jurkat and WTC-11 cell lines. Jurkat RNA was procured from Ambion (Thermo, PN AM7858) and WTC-11 RNA was extracted from WTC-11 cells (Coriell, GM25256). The RNA was analyzed on a Thermo Nanodrop UV-Vis and an Agilent Bioanalyzer to confirm the RNA concentration and ensure RNA integrity. From the RNA, cDNA was synthesised using the NEB Single Cell/Low Input cDNA Synthesis and Amplification Module (New England Biolabs).

Approximately $300 \mathrm{ng}$ of Jurkat CDNA or WTC-11 cDNA was converted into a SMRTbell library using the Iso-Seq Express Kit SMRT Bell Express Template prep kit 2.0 (Pacific Biosciences). This protocol employs bead-based size selection to remove low mass cDNA, specifically using an 86:100 bead-to-sample ratio (Pronex Beads, Promega). Library preparations were performed in technical duplicate. We sequenced each library on a SMRT cell on the Sequel II system using polymerase v2.1 with a loading concentration of $85 \mathrm{pM}$. A two-hour extension and 30 hour movie collection time was used for data collection. The 'ccs' command from the PacBio SMRTLink suite (SMRTLink version 9) was used to convert raw reads into Circular Consensus Sequence (CCS) reads. CCS reads with a minimum of three full passes and a 99\% minimum predicted accuracy (QV20) were kept for further analysis.

\section{Aligning IrRNA-seq data to a reference genome and BAM manipulation}

Full-length non-concatemers (FLNC) reads were aligned to the human genome of reference GRCh38.p13 [22] using minimap2 [23] (2.17-r941), and non-primary (secondary, supplementary, and unmapped) alignments were discarded by samtools [24] (1.9); a FLNC-alignment BAM file was generated. We used the GATK (4.1.9.0) function SplitNCigarReads (SNCR) to split reads at intronic regions, generating a second BAM file. We generated a third BAM file by correcting flags of the SNCR output BAM with flagCorrection (https://github.com/vladimirsouza//rRNAseqVariantCalling/blob/main/flagCorrection.r).

\section{Calling variants from IrRNA-seq with DeepVaraint}

From the flagCorrection output BAM file, genomic variants were called by DeepVariant (1.1.0), using the argument --model_type PACBIO. Variants with a QUAL score lower than 15 were filtered out. 


\section{Calling variants from IrRNA-seq with Clair3}

For SNP calling, from the unmanipulated FLNC-alignment BAM file, variants were called by Clair3 (v0.1-r5), using the argument --platform="hifi" and the pre-trained model downloaded from http://www.bio8.cs.hku.hk/clair3/clair3 models/clair3 models.tar.gz, and VCFTools (0.1.16) was used to keep only SNPs. For indel calling, from the flagCorrection output BAM file, Clair3 was run in the same way, and VCFTools was used to keep only indels. In both cases, we considered calls only from the pileup model by using the output file pileup.vcf.gz. The SNP- and indel-only VCF files were concatenated by bcftools (1.9) concat. For sites that culminated with two different variants (one SNP and one indel), we used our function removeRepeatedLowerQualSites.r (https://github.com/vladimirsouza/lrRNAseqVariantCalling/blob/main/tools/removeRepeatedL owerQualSites.r) to remove the variant with the lowest quality (QUAL) value.

\section{Calling variants from IrRNA-seq with GATK}

From the SNCR output BAM file, read groups were added to the BAM file by Picard [25] AddOrReplaceReadGroups function. Similarly to short-read data, variants were called with GATK's pipeline, which consisted of the following steps: generating recalibration table for base quality score recalibration (BQSR) with BaseRecalibrator; applying BQSR with ApplyBQSR; variant calling with HaplotypeCaller; consolidating and genotyping genomic variant call formats (GVCFs) with GenotypeGVCFs; and merging scattered phenotype VCF files with GatherVcfs. For variant-quality score recalibration (VQSR) and filtering, the GATK pipeline used was consisted of the following: VQSR and applying recalibration, both for SNPs and indels, with VariantRecalibrator and ApplyVQSR, respectively.

\section{Generating the ground truth VCFs for Jurkat and WTC-11 cells}

To generate the ground truth of SNPs and indels from Jurkat cells, two Illumina short-read DNA sequencing datasets [26] were downloaded in FASTQ format. The reads from both datasets were aligned to the human reference genome GRCh38.p13 with BWA-MEM [27]. Non-primary (secondary and supplementary) alignments were discarded and the two BAM files were merged by samtools. The same read group was assigned to all reads of the merged BAM by Picard AddOrReplaceReadGroups. Duplicate reads were marked by samtools fixmate followed by samtools markdup. Variants were called with GATK's pipeline, which consists of: generating recalibration table for base quality score recalibration (BQSR) with BaseRecalibrator; applying BQSR with ApplyBQSR; variant calling with HaplotypeCaller, with ploidy parameter set to diploid; consolidating and genotyping genomic variant call formats (GVCFs) with GenotypeGVCFs; and merging scattered phenotype VCF files with GatherVcfs. For variant-quality score recalibration (VQSR) and filtering, the GATK pipeline used was consisted of the following steps: VQSR and applying recalibration, both for SNPs and indels, with VariantRecalibrator and ApplyVQSR, respectively. 
The ground truth variants from WTC-11 cells (a VCF file) was downloaded from the Allen Institute [28]. To generate this VCF, 151 bp paired-end reads, at a mean depth of 100X, were aligned to GRCh38 using BWA-MEM (0.7.13). Duplicates were marked using Picard MarkDuplicates (2.3.0). The GATK's pipeline (3.5) used consisted of the following steps: local realignment around indels; BQSR; variants calling using HaplotypeCaller; and filtering using VQSR. We kept only variants from chromosomes chr1, ..., chr22, chrX, and chrY.

\section{Selecting high confident regions of the ground truth to compare the methods}

Since the read coverage of the Jurkat short-read DNA-seq data that we used is not high (overall coverage equal to $38 \mathrm{x}$ ), only variants residing in regions with short-read coverage higher than 20 reads were considered so as to avoid potential false positives (FPs) due to low short-read coverage. The variants that passed this coverage filter were considered to be the ground truth for the comparisons of variants called from Iso-Seq Jurkat data.

To avoid mapping/assembly errors (e.g., due to paralogous or repetitive regions), regions with short-read coverage higher than the 95th-percentile (98 reads) were also ignored. Moreover, to avoid other poorly-aligned regions (e.g., caused by missing regions of the genome) and after some manual investigation on IGV that highlighted some questionable alignments, any $201 \mathrm{bp}$ window that contains more than three variants was removed. And finally, only regions of the genome that had Iso-Seq coverage $>0$ were retained.

For the WTC-11 comparisons, a similar strategy was used. But, since the ground-truth VCF file was generated from high-coverage DNA-seq datasets, the arbitrary 20 reads as minimum coverage was not applied. Instead, the 5th-percentile (72 reads) was used as the minimum read coverage. The 95th-percentile (168 reads) was the maximum read coverage.

\section{Identifying sites that come from ASE genes}

RNA Illumina reads from WTC-11 cells were downloaded from the NCBI portal [29], identifiers GSM5330767, GSM5330768, and GSM5330769; and also from the ENCODE portal [30], identifiers ENCLB366GPZ, ENCLB122OCH, and ENCLB979NPE. STAR (2.7.0f) [31] was used to align the FASTQ files to the genome of reference GRCh38.p13; samtools (1.9) was used to remove secondary and supplementary alignments. GATK's ASEReadCounter function was used to calculate read counts per allele of the sites defined by our ground-truth VCF file for WTC-11. We ignored sites with RNA short-read and Iso-Seq coverage lower than 40 and 20 reads, respectively. From the table output by ASEReadCounter, a chi-squared goodness-of-fit test was applied, independently for each site, to test equal frequencies of reference and alternative alleles, and the p-values were corrected by the Benjamini-Hochberg multiple test correction. Sites with q-values lower than 0.05 were considered ASE sites. 


\section{Selecting indels within and outside homopolymer repeats}

Sites with Iso-Seq coverage lower than 20 reads were filtered out. To avoid poorly-aligned regions of Iso-Seq reads, any $201 \mathrm{bp}$ window that contains more than three variants (called by any tested pipeline) was removed. Only sites within $20 \mathrm{bp}$ of a splice junction were used. To avoid ambiguity in the classification of variant types, heterozygous-alternative variants were filtered out.

\section{Declarations}

\section{Ethics approval and consent to participate}

Ethics approval is not applicable to this work.

\section{Availability of data and materials}

The code used for all analyses presented in this paper, along with flagCorrection function, are available in the public github repository:

https://github.com/vladimirsouza/lrRNAseqVariantCalling (snapshot of repository at DOI:10.5281/zenodo.6011960). Important files generated for the analysis are available in a public zenodo repository (DOI: 10.5281/zenodo.5960321).

For all benchmarking analyses, several functions were created and are available as an $\mathrm{R}$ package in the public repository https://github.com/vladimirsouza/lrRNAseqBenchmark (snapshot at DOI: 10.5281/zenodo.6012005).

\section{Competing interests}

ET is an employee of Pacific Biosciences. All remaining authors declare that they have no competing interests.

\section{Funding}

MDR acknowledges support from the University Research Priority Program Evolution in Action at the University of Zurich. GMS acknowledges support from the National Institutes of Health (NIH) grant R35GM142647. VBCS acknowledges support from Conselho Nacional de 
Desenvolvimento Científico e Tecnológico. The funder played no role in the design of this study or in its execution.

\section{Authors' contributions}

VBCS, GS, ET, and MDR conceived the study. VBCS designed and implemented all code, ran all data analyses and wrote the manuscript. BJ contributed code and ideas for the analyses. MDR, GS, and ET interpreted data and supervised all analyses. GS, EAN, and $\mathrm{KKH}$ conducted experiments and made Jurkat and WTC-11 Iso-Seq data available. All authors revised the manuscript writing and approved the final manuscript.

\section{Acknowledgements}

The long-read sequencing was performed at the Maryland Genomics at the University of Maryland Institute for Genome Sciences. We acknowledge various Robinson lab members for helpful feedback on figures and analyses.

\section{References}

1. Koboldt DC. Best practices for variant calling in clinical sequencing. Genome Med. 2020;12: 91.

2. Sandmann S, de Graaf AO, Karimi M, van der Reijden BA, Hellström-Lindberg E, Jansen $\mathrm{JH}$, et al. Evaluating Variant Calling Tools for Non-Matched Next-Generation Sequencing Data. Sci Rep. 2017;7: 43169.

3. Mantere T, Kersten S, Hoischen A. Long-Read Sequencing Emerging in Medical Genetics. Front Genet. 2019;10: 426.

4. DePristo MA, Banks E, Poplin R, Garimella KV, Maguire JR, Hartl C, et al. A framework for variation discovery and genotyping using next-generation DNA sequencing data. Nat Genet. 2011;43: 491-498.

5. Narasimhan V, Danecek P, Scally A, Xue Y, Tyler-Smith C, Durbin R. BCFtools/RoH: a hidden Markov model approach for detecting autozygosity from next-generation sequencing data. Bioinformatics. 2016;32: 1749-1751.

6. Garrison E, Marth G. Haplotype-based variant detection from short-read sequencing. arXiv [q-bio.GN]. 2012. Available: http://arxiv.org/abs/1207.3907

7. Rimmer A, Phan H, Mathieson I, Iqbal Z, Twigg SRF, Wilkie AOM, et al. Integrating mapping-, assembly- and haplotype-based approaches for calling variants in clinical sequencing applications. Nat Genet. 2014;46: 912-918.

8. Wang B, Tseng E, Baybayan P, Eng K, Regulski M, Jiao Y, et al. Variant phasing and haplotypic expression from long-read sequencing in maize. Commun Biol. 2020;3: 78. 
bioRxiv preprint doi: https://doi.org/10.1101/2022.02.08.479579; this version posted February 10,2022 . The copyright holder for this preprint (which was not certified by peer review) is the author/funder, who has granted bioRxiv a license to display the preprint in perpetuity. It is made available under aCC-BY 4.0 International license.

9. Tardaguila M, de la Fuente L, Marti C, Pereira C, Pardo-Palacios FJ, Del Risco H, et al. SQANTI: extensive characterization of long-read transcript sequences for quality control in full-length transcriptome identification and quantification. Genome Res. 2018. doi:10.1101/gr.222976.117

10. Mudge JM, Harrow J. The state of play in higher eukaryote gene annotation. Nat Rev Genet. 2016;17: 758-772.

11. Rivas MA, Pirinen M, Conrad DF, Lek M, Tsang EK, Karczewski KJ, et al. Human genomics. Effect of predicted protein-truncating genetic variants on the human transcriptome. Science. 2015;348: 666-669.

12. Poplin R, Chang P-C, Alexander D, Schwartz S, Colthurst T, Ku A, et al. A universal SNP and small-indel variant caller using deep neural networks. Nat Biotechnol. 2018;36: 983-987.

13. Luo R. Clair3 - Integrating pileup and full-alignment for high-performance long-read variant calling. In: GitHub repository [Internet]. 2021 [cited 13 Sep 2021]. Available: https://github.com/HKU-BAL/Clair3

14. Ahsan MU, Liu Q, Fang L, Wang K. NanoCaller for accurate detection of SNPs and indels in difficult-to-map regions from long-read sequencing by haplotype-aware deep neural networks. Genome Biol. 2021;22: 261.

15. Edge $P$, Bansal V. Longshot enables accurate variant calling in diploid genomes from single-molecule long read sequencing. Nat Commun. 2019;10: 4660.

16. Shafin K, Pesout T, Chang PC, Nattestad M. Haplotype-aware variant calling enables high accuracy in nanopore long-reads using deep neural networks. bioRxiv. 2021. Available: https://www.biorxiv.org/content/10.1101/2021.03.04.433952v1.abstract

17. pbsv: PacBio structural variant (SV) calling and analysis tools. In: GitHub repository [Internet]. [cited 23 Sep 2021]. Available: https://github.com/PacificBiosciences/pbsv

18. Martin M, Patterson M, Garg S, Fischer S, Pisanti N. WhatsHap: fast and accurate read-based phasing. BioRxiv. 2016. Available:

https://www.biorxiv.org/content/10.1101/085050v1.abstract

19. Kuo RI, Cheng Y, Zhang R, Brown JWS, Smith J, Archibald AL, et al. Illuminating the dark side of the human transcriptome with long read transcript sequencing. BMC Genomics. 2020;21: 751.

20. Sahlin K, Tomaszkiewicz M, Makova KD, Medvedev P. Deciphering highly similar multigene family transcripts from Iso-Seq data with IsoCon. Nat Commun. 2018;9: 4601.

21. Logsdon GA, Vollger MR, Eichler EE. Long-read human genome sequencing and its applications. Nat Rev Genet. 2020;21: 597-614.

22. Human Genome Overview. In: Genome Reference Consortium [Internet]. [cited 19 Mar 2021]. Available: https://www.ncbi.nlm.nih.gov/grc/human

23. Li H. Minimap2: pairwise alignment for nucleotide sequences. Bioinformatics. 2018;34: 3094-3100.

24. Danecek P, Bonfield JK, Liddle J, Marshall J, Ohan V, Pollard MO, et al. Twelve years of SAMtools and BCFtools. Gigascience. 2021;10. doi:10.1093/gigascience/giab008 
25. Toolkit P. Picard toolkit. Broad Institute, Github Repository. 2019.

26. Gioia L, Siddique A, Head SR, Salomon DR, Su Al. A genome-wide survey of mutations in the Jurkat cell line. BMC Genomics. 2018;19: 334.

27. Li H, Durbin R. Fast and accurate short read alignment with Burrows-Wheeler transform. Bioinformatics. 2009;25: 1754-1760.

28. Allen Institute for Cell Science. Allen Institute for Cell Science WTC-11 short read whole genome sequence. [cited 16 Nov 2021]. Available:

https://open.quiltdata.com/b/allencell/tree/aics/wtc11_short_read_genome_sequence/

29. NCBI Resource Coordinators. Database resources of the National Center for Biotechnology Information. Nucleic Acids Res. 2018;46: D8-D13.

30. Davis CA, Hitz BC, Sloan CA, Chan ET, Davidson JM, Gabdank I, et al. The Encyclopedia of DNA elements (ENCODE): data portal update. Nucleic Acids Res. 2018;46: D794-D801.

31. Dobin A, Davis CA, Schlesinger F, Drenkow J, Zaleski C, Jha S, et al. STAR: ultrafast universal RNA-seq aligner. Bioinformatics. 2013;29: 15-21. 